\title{
Efectos del tadalafilo en el deseo sexual y la excitación/lubricación en mujeres postmenopáusicas
}

\section{Effects of Tadalafil on Sexual Desire and Arousal/Lubrication in Postmenopausal Women}

Franklin José Espitia De La Hoz 1,2,3,4®

${ }^{1}$ Director Científico, Hathor, Clínica Sexológica, Eje Cafetero, Colombia

Dirección para correspondencia Franklin José Espitia De La Hoz, MD,

${ }^{2}$ Ginecología y Obstetricia, Universidad Militar Nueva Granada, Bogota, Colombia

${ }^{3}$ Sexología Clínica, Master en Sexología: Educación y Asesoramiento Calle 30 Norte \# 20-10, Casa D3, Parque Residencial Los Ocobos, Armenia, Quindío, Colombia (e-mail: espitiafranklin@hotmail.com).

Sexual, Universidad de Alcalá de Henares, Madrid, España

${ }^{4}$ Uroginecología, FUCS (Hospital de San José)/Unicamp, Campinas, Brasil

Urol Colomb 2021;30(4):e231-e239.

\section{Resumen}

\section{Palabras Clave}

- disfunciones sexuales fisiológicas

- mujeres

- posmenopausia

- tadalafilo
Objetivo Determinar los efectos y la seguridad del tadalafilo en el deseo sexual y la excitación/lubricación en mujeres postmenopáusicas.

Métodos Estudio cuasi experimental (con comparación antes de la intervención y después), con seguimiento de la cohorte de pacientes: 228 mujeres mayores de 40 años en postmenopausia, que consultaron por bajo deseo sexual y alteración de la excitación /lubricación, en una clínica sexológica en Armenia (Colombia), entre 2018 y 2019. Las mujeres recibieron $2,5 \mathrm{mg}$ diarios de tadalafilo durante 12 semanas. Se determinaron las puntuaciones del instrumento, el Índice de Función Sexual Femenina (IFSF), cada cuatro semanas.

Resultados El promedio de edad fue de $54,36 \pm 4,92$ años. Antes del tratamiento, la media en la puntuación del IFSF fue de 22,41 puntos (intervalo de confianza del $95 \%$ [IC95\%]: 7,11-29,76); a las 4 semanas, 24,73 puntos (IC95\%: 23,51-30,28); a las 8 semanas, 25,97 puntos (IC95\%: 24,26-31,53); y, al final, 26,99 puntos (IC95\%: 25,97$32,46)$, con diferencias estadísticamente significativas entre las 4 medidas pareadas $(p<0,001)$. La excitación fue el dominio con mayor puntuación tras el tratamiento (4,83 puntos; IC 95\%: 4,36-4,91), seguida por la lubricación (4,77 puntos; IC95\%: 4,284,87). El deseo sexual mostró un significativo incremento al final del estudio $(4,29$ puntos; IC95\%: 4,07-4,39). Se presentaron efectos adversos leves. Se observó una satisfacción con el consumo de tadalafilo del $84,21 \%$.

Conclusiones El tratamiento diario con $2,5 \mathrm{mg}$ de tadalafilo genera mejoría en la función sexual de mujeres en la postmenopausia, y el incremento en la puntuación del IFSF demuestra su efecto positivo. recibido

24 de agosto de 2020

aceptado

19 de agosto de 2021
DOI https://doi.org/ $10.1055 / \mathrm{s}-0041-1740343$ ISSN 0120-789X. e ISSN 2027-0119.
(C) 2021. Sociedad Colombiana de Urología. All rights reserved. This is an open access article published by Thieme under the terms of the Creative Commons Attribution-NonDerivative-NonCommercial-License, permitting copying and reproduction so long as the original work is given appropriate credit. Contents may not be used for commercial purposes, or adapted, remixed, transformed or built upon. (https://creativecommons.org/ licenses/by-nc-nd/4.0/)

Thieme Revinter Publicações Ltda., Rua do Matoso 170, Rio de Janeiro, RJ, CEP 20270-135, Brazil 


\begin{abstract}
Keywords

- sexual dysfunction, physiological

- women

- postmenopause

- tadalafil

Objective To determine the effects and safety of tadalafil on sexual desire and arousal/lubrication in postmenopausal women.

Materials and methods Quasi-experimental study (with a comparison before and after the intervention) with follow-up of the patient cohort: 228 postmenopausal women older than 40 years of age, who consulted due to low sexual desire and altered arousal/lubrication, in a sexology clinic in Armenia (Colombia) between 2018 and 2019. The women received $2.5 \mathrm{mg}$ of tadalafil daily for 12 weeks. The scores on the instrument used, the Female Sexual Function Index, (FSFI), were determined every four weeks.

Results The mean age was $54.36 \pm 4.92$ years. Before the treatment, the mean FSFI score was of 22.41 points ( $95 \%$ confidence interval [ $95 \% \mathrm{Cl}$ ]: $7.11-29.76$ ); at 4 weeks, it was of 24.73 points ( $95 \% \mathrm{Cl}: 23.51-30,28)$; at 8 weeks, 25.97 points ( $95 \% \mathrm{Cl}: 24.26-$ $31.53)$; and, at the end, it was of 26.99 points ( $95 \% \mathrm{Cl}: 25.97-32.46)$, with statistically significant differences among the four paired measurements $(p<0.001)$. Arousal was the domain with the highest posttreatment score (4.83 points; $95 \% \mathrm{Cl}: 4.36-4.91)$, followed by lubrication (4.77 points; $95 \% \mathrm{Cl}$ : 4.28-4.87). Sexual desire showed a significant increase at the end of the study (4.29 points; $95 \% \mathrm{Cl}: 4.07-4.39)$. Mild adverse effects occurred. A rate of satisfaction of $84.21 \%$ with the intake of tadalafil. Conclusions The daily treatment with tadalafil $2.5 \mathrm{mg}$ results in an improvement in sexual function in postmenopausal women; the increase in the score on the FSFI demonstrates its positive effect.
\end{abstract}

\section{Introducción}

Las mujeres, más que los hombres, se ven afectadas por algún tipo de trastorno sexual a lo largo de la vida. En Latinoamérica, los datos reportan la presencia de trastornos sexuales femeninos en los siguientes porcentajes: 21\% (Cartagena de Indias), 33,2\% (La Habana), 40,9\% (Ciudad de Panamá), 94,5\% (Montevideo) y 98,5\% (Quito). ${ }^{1,2}$ La prevalencia de disfunción sexual en mujeres colombianas fue informada por Espitia-De La $\mathrm{Hoz}^{3}$ en $32,97 \%$; este autor describe dificultades con el deseo (32,97\%), el orgasmo (21,93\%), la excitación (16,86\%), la lubricación (14,79\%), y el dolor (7,56\%).

A la fecha, existen múltiples agentes farmacológicos utilizados para el tratamiento de las disfunciones sexuales femeninas; no obstante, la mayoría tiene indicaciones no aprobadas. De hecho, se carece de suficientes estudios controlados, de terapias medicamentosas, para el tratamiento de las disfunciones sexuales en mujeres postmenopáusicas. ${ }^{4}$

Las fosfodiesterasas (FDEs) son enzimas que hidrolizan los nucleótidos cíclicos: inactivan al adenosín monofosfato cíclico (AMFc) y al guanosín monofosfato cíclico (GMFc), los segundos mensajeros de la prostaciclina (o prostaglandina I2, PGI2) y del óxido nítrico (ON), respectivamente, los que promueven vasorrelajación. Entre las FDEs, existen 11 isoenzimas (FDE-1 a FDE-11). La FDE-5 es la enzima que cataliza el deterioro de GMFc, pero puede ser inhibida por los inhibidores orales de FDE-5 (IFDE-5), como el sildenafil, el tadalafil y el vardenafil, produciendo relajación del músculo liso e influjo de sangre. ${ }^{5,6}$
La FDE-5 se expresa en diferentes tejidos (vasculaturas arterial coronaria y venosa, músculo esquelético, músculos liso visceral y traqueo-bronquial), y también en las plaquetas, el fibroblasto de pulmón, y el cerebelo. ${ }^{6}$

En la actualidad, los IFDE-5 se consideran la primera línea en el tratamiento de la disfunción eréctil (DE), debido a su alta eficacia, buena tolerabilidad y facilidad de administración. $^{7}$

El tadalafilo es un potente y selectivo IFDE-5, aprobado por la Food and Drug Administration (FDA) y la Agencia Europea de Medicamentos (European Medicines Agency, EMA, en inglés), para el tratamiento de la $\mathrm{DE} ;^{8}$ además, tiene efecto antihipertensivo en sujetos con hipertensión no controlada, con un adicional beneficio de disminuir la mortalidad y la morbilidad. ${ }^{9}$

La postmenopausia se ha definido como el periodo que se inicia a partir del año de transcurrida la menopausia, extendiéndose hasta el final de la vida. ${ }^{10}$ En esta etapa de la vida de la mujer, ocurren muchos cambios fisiológicos que podrían tener influencia en la aparición de disfunciones sexuales. $^{11}$

El éxito alcanzado con los IFDE-5 en el tratamiento de la disfunción de la excitación sexual masculina ha alentado su investigación para mejorar la congestión genital y la lubricación vaginal de las mijeres. ${ }^{12}$

El tadalafilo se absorbe por vía oral, y alcanza la concentración plasmática máxima media a las 4 horas, con una vida media de eliminación de 17,5 horas, pero con una duración de acción entre 24 y 36 horas, lo que hace posible su posología como monodosis diaria. ${ }^{9,13}$ 
Anexo 1 Índice de Función Sexual Femenina (IFSF)

\begin{tabular}{|c|c|c|c|c|c|}
\hline & Preguntas & Puntaje & Factor & Mínimo & Máximo \\
\hline Deseo & $1-2$ & $1-5$ & 0,6 & 1,2 & 6 \\
\hline Excitación & $3-6$ & $0-5$ & 0,3 & 0 & 6 \\
\hline Lubricación & $7-10$ & $0-5$ & 0,3 & 0 & 6 \\
\hline Orgasmo & $11-13$ & $0-5$ & 0,4 & 0 & 6 \\
\hline Satisfacción & $14-16$ & $0-5$ & 0,4 & 0,8 & 6 \\
\hline \multirow[t]{2}{*}{ Dolor } & $17-19$ & $0-5$ & 0,4 & 0 & 6 \\
\hline & & & Total & 2 & 36 \\
\hline
\end{tabular}

Así como los IFDE-5 han revolucionado el tratamiento de la $\mathrm{DE}^{8,9,12}$ en los hombres, al conceder altas tasas de efectividad y seguridad, facilitando su uso de forma rutinaria, en las mujeres se han reportado mejoras en los parámetros de eficacia en las disfunciones sexuales. ${ }^{10}$

En concordancia con los datos acumulados, los cuales indican que las dosis bajas o el uso a largo plazo de tadalafilo, pueden proporcionar beneficios potenciales adicionales y/o mejores respuestas al tratamiento, ${ }^{12,13}$, los que sumados a su vida media prolongada (debido a las propiedades farmacocinéticas), ${ }^{8,9,13}$ le permiten al tadalafilo, la adaptación a un régimen diario de dosificación, con la ventaja adicional de separar la medicación de los encuentros sexuales, facilitando la espontaneidad de la actividad sexual. ${ }^{13}$ Es por eso, que, en este estudio, con la dosis de 2,5 mg de taladafilo, se buscó determinar su seguridad, tolerabilidad, la satisfacción de las pacientes, y sus efectos en el deseo sexual y en la excitación/lubricación, en mujeres postmenopáusicas en el Quindío, Colombia.

\section{Métodos}

Diseño de estudio y población. Estudio cuasi experimental (comparación antes de la intervención y posterior a esta/ diseño pretest/postest) en mujeres mayores de 40 años con menopausia no inferior a 1 año, pertenecientes al programa de Menopausia y Climaterio, que consultaron entre el 1er de octubre de 2017 y el 31 de octubre 2018, por bajo deseo sexual y alteración de la excitación/;lubricación, en una clínica sexológica de referencia en Armenia, Colombia. No se consideró el cegamiento ni la asignación de un grupo de control. Se utilizó como instrumento el Índice de Función Sexual Femenino (IFSF). Se excluyeron las mujeres con antecedente de histerectomía, ooforectomía, las que no recordaban la fecha de la última menstruación, las que estaban recibiendo o habían recibido terapia de remplazo hormonal (sistémica o local) en los últimos tres meses previos al estudio, las que no tenían pareja, antecedente personal de cáncer, patologías psiquiátricas diagnosticadas, y las que no firmaron el consentimiento informado.

El tamaño de la muestra se calculó con base en la población del programa de Menopausia y Climaterio, y se obtuvo una muestra de 216 participantes, la cual se ajustó hasta 240 mujeres ante una eventual pérdida del $10 \%$. Se hizo un muestreo consecutivo.

\section{Procedimiento}

Las mujeres candidatas al estudio fueron identificadas por el médico sexólogo durante la atención sexológica. Una enfermera profesional, diplomada en salud sexual, integrante del equipo de investigación, y dos auxiliares de enfermería verificaban que la paciente cumpliera con los criterios de selección. Le explicaban el objetivo del estudio, y solicitaban la firma del consentimiento informado; posteriormente, mediante entrevista cara a cara, completaban los datos sociodemográficos y de salud sexual y reproductiva, y, finalmente, le entregaban el IFSF.

El IFSF es un cuestionario multidimensional y autoadministrado de 19 preguntas, con 6 dominios para la evaluación y cuantificación de la función sexual femenina: deseo (ítems 1-2), excitación, ${ }^{3-6}$ lubricación, ${ }^{7-10}$ orgasmo, ${ }^{11-13}$ satisfacción, ${ }^{14-16}$ y dolor. ${ }^{17-19}$ El rango de la puntuación para los ítems 3 a 14 y 17 a 19 oscila entre 0 y 5; para los ítems $1,2,15$ y 16 , entre 1 y 5 . El rango del puntaje de la puntuación total oscila entre 2 y 36 puntos $^{14}$ (-Anexo 1). Ha sido validado en español, y, inicialmente mostró una buena consistencia interna para todos los dominios en una muestra de la población general, así como en subgrupos de pacientes mujeres con disfunción sexual, con características psicométricas adecuadas (alfa de Cronbach para cada dimensión entre 0,82 y 0,97). La fiabilidad test-retest es aceptable $(r=0,79-0,86)$. Los puntajes más altos indican una mejor función sexual. Los resultados de la evaluación con valores inferiores a 26,55, o cuando la puntuación de algún dominio es menor a 3,6 puntos, sugieren la presencia de disfunción sexual. ${ }^{3,15,16}$ Es considerado el patrón oro para evaluar la función sexual femenina. ${ }^{17}$

A las participantes se les hizo seguimiento clínico cada 4 semanas, hasta completar las 12 semanas, para evaluar tanto los efectos como la seguridad y la continuación del medicamento. Se les evaluaba el IFSF para establecer la comparación de la puntuación de cada uno de los dominios y de la puntuación total en cada seguimiento.

Intervención. La dosis de tadalafilo fue de $2,5 \mathrm{mg}$ diarios durante 12 semanas.

La seguridad fue evaluada mediante el registro y la valoración de la incidencia de reacciones adversas. Una reacción adversa se clasificó como: leve: cefalea, congestión nasal, dolor de espalda, náuseas, y rubefacción (enrojecimiento de la cara); moderada: hemorragia nasal, 
lipotimia, palpitaciones, visión borrosa, y vómitos;- y severa: convulsiones, hematuria, muerte súbita, pérdida pasajera de la memoria, y urticaria.

La satisfacción fue evaluada por meio de la relación entre el efecto terapéutico y la presencia de efectos adversos, con una escala de impresión cualitativa que contempló las alternativas: satisfecha: $\geq 75 \%$; medianamente satisfecha: 51-74\%; e insatisfecha: $\leq 50 \%$.

La tolerabilidad se evaluó mediante una escala de impresión cualitativa que contempló las siguientes alternativas: excelente: ninguna reacción adversa medicamentosa;buena: la reacción adversa medicamentosa no interfiere de forma significativa con la actividad de la paciente;regular: la reacción adversa medicamentosa interfiere de forma significativa con la actividad normal de la paciente; y mala: la reacción adversa medicamentosa sobrepasa el efecto terapéutico.

Variables medidas. Se midieron las variables sociodemográficas (edad, etnia, nivel de escolaridad, estrato socioeconómico, estado civil, ocupación, afiliación al sistema general de seguridad social en salud, religión, y área de residencia); peso, talla, índice de masa corporal (IMC); salud sexual y reproductiva (edad de la menarquia, embarazos, paridad, edad de la menopausia, y tiempo en postmenopausia); comportamiento sexual (orientación sexual, edad de la primera relación sexual, coito - vaginal o anal-, sexo oral, promedio mensual de relaciones sexuales, número de parejas sexuales, tiempo de convivencia en pareja, uso de juguetes eróticos, disfunciones sexuales en la pareja, e infidelidad); antecedentes personales; hábitos (tabaquismo y ingesta de alcohol). Además, se hicieron las preguntas de los dominios del IFSF. Se evaluaron los efectos del tadalafilo, su seguridad, y la tolerancia de las pacientes, y se determinó la incidencia de efectos secundarios. La información se digitó en el programa Microsoft Excel (Microsoft Corp., Redmond, WA, EEUU), versión 14.0, y también se utilizó el programa Statistical Package for the Social Sciences (IBM SPSS Statistics, IBM Corp., Armonk, NY, EEUU), versión 24.0.

Análisis estadístico. Se realizó un análisis descriptivo de las variables categóricas sociodemográficas. Se calcularon frecuencias absolutas, porcentajes e intervalos de confianza del 95\% (IC95\%) para variables categóricas, y media y desviación estándar, mediana o rango, mínimo y máximo e IC95\%, según la distribución de las variables continuas. Se realizó una medición de diferencia de medias con su correspondiente IC95\%. En la comparación de promedios, de acuerdo a la homogeneidad o no de las varianzas, se utilizaron pruebas pareadas como $t$ de Student o Wilcoxon, y no pareadas como $t$ de Student o U de Mann-Whitney, respectivamente. La comparación de porcentajes de mejoría fue evaluada con las pruebas de chi cuadrado o exacta de Fisher. Se consideró significancia estadística cuando $p<0,05$.

Aspectos éticos. El estudio fue aprobado por el comité de ética de la institución participante. Se garantizó la confidencialidad, el anonimato y la privacidad de la información. Se tuvo en cuenta la Declaración de Helsinki, en la que se establecen los principios éticos para las investigaciones médicas en seres humanos. Se consideró la Resolución 8.430 de 1993 del Ministerio de Salud de la República de Colombia, la cual determina las normas científicas, técnicas y administrativas para la investigación en salud en la nación.

\section{Resultados}

Se reclutaron en total 240 participantes, y se excluyeron 12 porque no asistieron a la evaluación final, por lo que el estudio se hizo con 228 mujeres.

La mayoría de las participantes eran hispánicas (70,61\%), casadas (46,92\%), amas de casa (39,91\%), de estrato socioeconómico medio (57,45\%), residentes en zona urbana (85,96\%), bachilleres (35,52\%), y católicas (90,35\%). El 78,94\% pertenecían al régimen contributivo de seguridad social en salud. Las variables sociodemográficas se describen en la -Tabla 1. La media del IMC fue de $26,23 \mathrm{~kg} / \mathrm{m}^{2}$, lo que indica que se trató de una población con sobrepeso.

Tabla 1 Características sociodemográficas de mujeres postmenopáusicas en el Quindío, 2017-2018

\begin{tabular}{|c|c|c|}
\hline Variables & $n=228$ & $\%$ \\
\hline Edad, años & $54,36 \pm 4,92$ & \\
\hline Peso $(\mathrm{kg})$ & 67,92 & \\
\hline Talla (m) & 1,61 & \\
\hline Índice de Massa Corporal $\left(\mathrm{kg} / \mathrm{m}^{2}\right)$ & 26,23 & \\
\hline \multicolumn{3}{|l|}{ Raza } \\
\hline Hispánica & 161 & 70,61 \\
\hline Afrodescendiente & 47 & 20,61 \\
\hline Indígena & 20 & 8,77 \\
\hline \multicolumn{3}{|l|}{ Estado civil } \\
\hline Soltera & 8 & 3,5 \\
\hline Casada & 107 & 46,92 \\
\hline Unión libre & 88 & 38,59 \\
\hline Viuda & 25 & 10,96 \\
\hline \multicolumn{3}{|l|}{ Nivel educativo } \\
\hline Primaria & 11 & 4,82 \\
\hline Secundaria & 81 & 35,52 \\
\hline Tecnológico & 74 & 32,45 \\
\hline Universitario & 62 & 27,19 \\
\hline \multicolumn{3}{|l|}{ Ocupación } \\
\hline Ama de casa & 91 & 39,91 \\
\hline Empleada & 103 & 45,17 \\
\hline Desempleada & 15 & 6,57 \\
\hline Pensionada & 19 & 8,33 \\
\hline \multicolumn{3}{|l|}{ Estrato socioeconómico } \\
\hline Alto & 56 & 24,56 \\
\hline Medio & 131 & 57,45 \\
\hline Bajo & 41 & 17,98 \\
\hline
\end{tabular}


El promedio de edad de las participantes fue de $54,36 \pm 4,92$ años, con un mínimo de 42 y un máximo de 69 años, y el promedio de edad la de la pareja fue de $60,27 \pm 7,41$ años.

El 55,26\% presentó la menopausia, en promedio, a los $49,17 \pm 4,23$ años. El $68,42 \%$ reportó haber tenido la última menstruación, en promedio, 8,37 $\pm 3,72$ años antes de la fecha del estudio. El 44,73\% refirió que nunca había recibido terapia de reemplazo hormonal.

Respecto a los hábitos, el 18,85\% eran fumadoras habituales, y el 84,64\% consumían alcohol de manera social.

Respecto a los antecedentes de salud sexual y reproductiva, la paridad presentó una mediana de 4 hijos en, con rango entre 0 y 15 hijos. El 68,85\% de las mujeres afirmaron haber tenido más de 3 embarazos.

La orientación sexual de las participantes era de predominio heterosexual $(93,42 \%)$, y la edad del inicio de la actividad sexual fue a los $16,35 \pm 2,13$ años. La práctica sexual más frecuente era el coito vaginal (100\%), y la menos usual, el coito anal (21,05\%); la masturbación fue considerada una práctica poco usual para el 69,29\% de las mujeres participantes. El 15,35\% manifestó el uso de juguetes sexuales, mientras que el $32,45 \%$ refirió que la pareja se negaba a utilizarlos. El 41,66\% fue enfática al afirmar que nunca los utilizaría.

En relación a los comportamientos sexuales, el número de parejas sexuales arrojó una mediana de 6 , con rango entre $1 \mathrm{y}$ 12. El 36,84\% afirmó tener más de 20 años de convivencia en pareja, y el 71,92\% enfatizó que la pareja presentaba alguna disfunción sexual. El 40,78\% aseguró que la pareja le había sido infiel por lo menos en una ocasión, y el 14,47\% afirmó haber sido infiel por lo menos en una ocasión.

El 29,82\% manifestó que practicaba actividad sexual por insistencia de la pareja, y el 49,12\%, para evitar conflictos con la pareja, mientras que el $21,05 \%$, por iniciativa propia. El $56,57 \%$ de las mujeres señalaran que, si dependiera de ellas, no volverían a tener, nunca más actividad sexual. El total de las mujeres refirieron tener actividad sexual mensual de forma ocasional, con una mediana de 3 veces al mes, y rango entre 0 y 5 .

El 9,64\% de las mujeres afirmó haber sufrido alguna forma de violencia sexual a lo largo de la vida, mientras que el 6,14\% manifestó abuso sexual por parte de la pareja.

El 74,56\% de las mujeres presentaban la disfunción sexual, en promedio, hacía 5,82 $\pm 1,77$ años.

Las comorbilidades más frecuentes fueron: dislipidemias (28,07\%), enfermedades cardiovasculares (25,43\%), e hipotiroidismo (14,91\%) (-Tabla 2).

Al comienzo del estudio (antes del tratamiento), en la población total, el promedio del IFSF fue de 22,41 puntos (IC95\%: 7,11-29,76), y, al final, fue de 26,99 (IC95\%: 9,79$32,59)$, siendo la mejoría estadísticamente significativa $(p<0,0001)$. Al inicio del estudio, el dominio con el menor puntaje fue el deseo (3,28 puntos; IC95\%: 3,17-3,52), cuyo puntaje al final fue de 4,29 (IC95\%: 4,07-4,39; $p<0,001$ ); y el de mayor puntaje fue el dolor (4,59 puntos; IC95\%: 3,494,78). En la - Tabla 3 se muestra el análisis descriptivo de los dominios del IFSF a lo largo del estudio. Las variaciones de las
Tabla 2 Comorbilidades en mujeres postmenopáusicas tratadas con tadalafilo $(n=228)$ en el Quindío, 2017-2018

\begin{tabular}{|l|l|l|}
\hline Morbilidad & $\mathbf{n}$ & \% \\
\hline Artritis reumatoide & 5 & 2,19 \\
\hline Depresión & 31 & 13,59 \\
\hline Diabetes & 23 & 10,08 \\
\hline Dislipidemias & 64 & 28,07 \\
\hline Enfermedad pulmonar obstructiva crónica & 15 & 6,57 \\
\hline Enfermedades cardiovasculares & 58 & 25,43 \\
\hline Hipotiroidismo & 34 & 14,91 \\
\hline Osteoporosis & 33 & 14,47 \\
\hline
\end{tabular}

puntuaciones medias, obtenidas antes y después de la ingesta del fármaco, presentaron diferencias estadísticamente significativas a lo largo del tiempo.

A las 4 semanas, la media basal del IFSF fue de 23,07 puntos (IC95\%: 8,53-29,88); a las 8 semanas, 25,42 puntos (IC95\%: 9,41-30,27); y, al final del tratamiento, 26,99 puntos (IC95\%: 9,79-32,59), diferencia estadísticamente significativa entre los 4 puntajes pareados $(p<0,001)$. Se observaron diferencias significativas entre los 4 puntajes $(p<0,001)$, entre los puntajes iniciales y a las 4 y 8 semanas $(p<0,001)$, entre los puntajes iniciales y finales ( $p$ $<0,001)$, y entre los puntajes a las 4 y 8 semanas y los finales $(p=0,027)$, lo que representó un incremento del $51,31 \%$ en la puntuación total del IFSF, por encima de 26,55 puntos respecto al inicio $(p<0,001)$.

La excitación fue el dominio con mayor puntuación posterior al tratamiento (4,83 puntos; IC95\%: 4,36-4,91), seguida por la lubricación (4,77 puntos; IC95\%: 4,28-4,87); esta mejoría representó un incremento del $85,52 \%$ y del $77,63 \%$, por encima de 3,6 puntos en dichos dominios, respectivamente. El deseo sexual mostró un incremento significativo al final del estudio $(p=0,0057)$ (-Tabla 3), lo que representó un incremento del 69,73\% por encima del 3,6 en la puntuación del dominio.

La mejoría en la puntuación del IFSF, a expensas del incremento en los dominios excitación y lubricación, se acompañó también de una mejoría significativa en la función orgásmica $(p=0,006)$.

En el dominio orgasmo, se observó que hubo mejoría en la puntuación, que pasó de 3,85 puntos (IC95\%: 3,72-3,94), al comienzo, a 4,35 puntos al final (IC95\%: 4,15-4,52; $p<0,001)$. Esto representó una recuperación del 57,89\% al $80,26 \%$ en la puntuación, por encima de 3,6 puntos en dicho dominio (-Tabla 3).

La satisfacción sexual se incrementó al final del estudio, y pasó del $65,78 \%$ a $94,73 \%(p<0,001)$, mientras que el dolor disminuyó del 16,22\% al 5,26\% ( $p<0,001)$ ( - Tabla 3).

Se observó que las mujeres que tenían actividad sexual 5 o más veces al mes tuvieron un promedio de IFSF mayor que las que tuvieron menos de 5 relaciones sexuales por mes $(p=0,021)$. El deseo sexual y la excitación/lubricación tuvieron una mayor puntuación en aquellas mujeres que 
Tabla 3 Análisis descriptivo de los dominios del Índice de Función Sexual Femenina en mujeres postmenopáusicas tratadas con tadalafilo $(n=228)$ en el Quindío, 2017-2018

\begin{tabular}{|c|c|c|c|}
\hline Dominio & Seguimiento & Puntuación & Intervalo de confianza del 95\% \\
\hline \multirow[t]{4}{*}{ Deseo } & Basal & 3,28 & $3,17-3,52$ \\
\hline & A las 4 semanas & 3,47 & $3,35-3,61$ \\
\hline & A las 8 semanas & 4,05 & $3,78-4,12$ \\
\hline & A las 12 semanas & 4,29 & $4,07-4,39$ \\
\hline \multirow[t]{4}{*}{ Excitación } & Basal & 3,32 & $3,24-3,49$ \\
\hline & A las 4 semanas & 3,55 & $3,41-3,68$ \\
\hline & A las 8 semanas & 4,17 & $3,76-4,35$ \\
\hline & A las 12 semanas & 4,83 & $4,36-4,91$ \\
\hline \multirow[t]{4}{*}{ Lubricación } & Basal & 3,41 & $3,35-3,61$ \\
\hline & A las 4 semanas & 3,68 & $3,57-3,89$ \\
\hline & A las 8 semanas & 4,29 & $3,65-4,41$ \\
\hline & A las 12 semanas & 4,77 & $4,28-4,87$ \\
\hline \multirow[t]{4}{*}{ Orgasmo } & Basal & 3,85 & $3,72-3,94$ \\
\hline & A las 4 semanas & 3,92 & $3,85-3,97$ \\
\hline & A las 8 semanas & 4,26 & $3,92-4,37$ \\
\hline & A las 12 semanas & 4,35 & $4,15-4,52$ \\
\hline \multirow[t]{4}{*}{ Satisfacción } & Basal & 3,96 & $3,83-4,17$ \\
\hline & A las 4 semanas & 4,11 & $4,04-4,22$ \\
\hline & A las 8 semanas & 4,43 & $4,11-4,58$ \\
\hline & A las 12 semanas & 4,56 & $4,42-4,63$ \\
\hline \multirow[t]{4}{*}{ Dolor } & Basal & 4,59 & $3,49-4,25$ \\
\hline & A las 4 semanas & 4,34 & $3,29-4,55$ \\
\hline & A las 8 semanas & 4,22 & $4,19-4,54$ \\
\hline & A las 12 semanas & 4,11 & $4,06-4,42$ \\
\hline \multirow[t]{4}{*}{ Total } & Basal & 22,41 & $7,11-29,76$ \\
\hline & A las 4 semanas & 23,07 & $8,53-29,88$ \\
\hline & A las 8 semanas & 25,42 & $9,41-30,27$ \\
\hline & A las 12 semanas & 26,99 & $9,79-32,59$ \\
\hline
\end{tabular}

tenían relaciones sexuales por iniciativa propia, en comparación con las que tenían actividad sexual por insistencia de la pareja $(p=0,018)$. Las mujeres que reportaron como satisfactoria su vida sexual arrojaron una puntuación mayor en el IFSF frente a las que la refirieron como insatisfactoria $(p=0,003)$.

Las participantes que tuvieron un mayor puntaje en el dominio deseo sexual tuvieron un puntaje menor en el dominio dolor y un puntaje mayor en los dominios excitación y lubricación que aquellas con menor deseo sexual, lo que fue estadísticamente significativo para cada dominio mencionado $(p=0,027 ; p=0,051 ; \mathrm{y} p=0,0057$, respectivamente).

El deseo o interés sexual se incrementó mucho menos en mujeres con exclusiva actividad sexual vaginal frente a las que tenían otras prácticas sexuales (vaginal, oral y/o anal), con diferencia estadísticamente significativa $(p=0,054)$.

Se observó que las mujeres con 5 o más años de postmenopausia, reportaron una menor puntuación en los dominios excitación y lubricación en comparación con aquellas con menos de 5 años de postmenopausia $(p=0,051)$.

El tadalafilo demostró efectos positivos en la función sexual de las mujeres en postmenopausia, pues las participantes experimentaron una mejoría significativa tanto en la excitación/lubricación como en el deseo sexual $(p=0,0001)$ y en otros dominios del IFSF. La frecuencia coital mensual, al final del estudio, alcanzó una mediana de 7 (rango: 3 a 12).

Respecto a la seguridad, se observó que la intensidad clínica de las reacciones adversas se ubicó en leve, y no se 
requirió tratamiento o suspensión de la medicación. Los efectos adversos reportados fueron: cefalea (6,14\%), enrojecimiento de la cara (4,82\%), congestión nasal (3,07\%), y náuseas $(2,19 \%)$.

De las 228 participantes, 192 tuvieron la impresión de que la medicación proporciona un importante efecto terapéutico sin eventos adversos, y la tasa de satisfacción fue del $84,21 \%$.

Respecto a la tolerabilidad del tratamiento, el $82,45 \%$ de las participantes la calificaron como buena.

\section{Discusión}

Se observan grandes diferencias en la prevalencia de disfunciones sexuales entre países, lo cual puede reflejar la influencia tanto de factores médicos como psicológicos, particularmente en el contexto de posibles diferencias socioeconómicas, culturales, y raciales; de igual manera, también existe la posibilidad de que influya la definición utilizada para cada disfunción, el tipo de ensayo realizado (cuestionario autoaplicable, entrevista por teléfono, cuestionario enviado por correo, entrevista personal etc.), y la característica de las muestras (población general versus pacientes de clínicas de sexualidad) estudiadas. ${ }^{3,4}$

El tadalafilo es una sustancia que se ha utilizado ampliamente en ambos sexos para el tratamiento de varias patologías. Su eficacia se ha estudiado en especial en los síntomas del tracto urinario inferior, ${ }^{18,19}$ debido a sus efectos metabólicos y microvasculares sobre el músculo esquelético y el tejido adiposo en pacientes con diabetes tipo $2,{ }^{20}$ así como para el tratamiento de la hipertensión pulmonar. ${ }^{21} \mathrm{Su}$ uso en mujeres parece mejorar aspectos sexuales, pero los estudios $^{22,23}$ han reportado algunas limitaciones como el tamaño pequeño de la muestra, la ausencia de un grupo de control, así como la falta de evaluación objetiva del tipo de disfunción sexual.

En general, las características sociodemográficas de la población participante en esta investigación se ajustan a las observadas (en población colombiana) por Espitia-De La Hoz ${ }^{11,16}$ en recientes estudios publicados sobre la presencia de disfunciones sexuales en mujeres en climaterio.

Nuestros resultados indican que el tadalafilo es una molécula con una elevada efectividad en el tratamiento de los trastornos de la excitación/lubricación, así como en el tratamiento del bajo deseo sexual $(p=0,0001)$; esto se asocia al hecho de que los IFDE-5 pueden disminuir el catabolismo del GMF cíclico, lo que ocasiona la relajación del clítoris y del músculo liso vascular vaginal, y un aumento en el flujo sanguíneo local. ${ }^{22}$

En el estudio de Caruso et al. ${ }^{23}$ en una población de 33 mujeres premenopáusicas con diabetes tipo 1 , afectadas por trastorno de la excitación sexual, estas fueron tratadas $5 \mathrm{mg}$ de tadalafilo diarios durante 12 semanas; los autores concluyeron que hubo mejoría del aspecto sexual subjetivo en la población participante.

Si bien se ha informado poca evidencia sobre la eficacia del tadalafilo en el tratamiento de las disfunciones sexuales femeninas, especialmente en mujeres tratadas con medicamentos antidepresivos ${ }^{24} \mathrm{o}$ afectadas por diabetes mellitus (DM) tipo 1 con trastornos de excitación, ${ }^{23}$ debemos resaltar que el $13,59 \%$ de la población participante en esta investigación presentaba dichas condiciones.

La prolongada duración de la eficacia del tadalafilo ${ }^{25}$ puede contribuir a que la paciente perciba la relación sexual con mayor naturalidad, lo que incrementa la disposición a la espontaneidad en las relaciones sexuales y reduce las presiones psicológicas que influyen de forma negativa en el funcionamiento sexual; ${ }^{26}$ esto puede tener un efecto positivo al asociarse a un incremento en la calidad de vida de la paciente.

Las publicaciones de algunos investigadores ${ }^{27}$ han informado resultados positivos con el uso de IFDE-5, en especial con el tratamiento con sildenafil; ellos documentan que los IFDE-5 podrían ser eficaces en muchos tipos de disfunciones sexuales femeninas, con mejoría en diferentes aspectos de la función sexual de la mujer, como el deseo sexual, la excitación, el orgasmo, y la satisfacción sexual. ${ }^{27}$ Los hallazgos son concordantes con los resultados de la presente investigación, en la que la excitación y la lubricación tuvieron una mejoría del $85,52 \%$ y 77,63, respectivamente, mientras el deseo sexual representó un incremento del 69,73\%, pudiéndose asociar dicha mejoría al aumento de los procesos excitatorios, útiles en mujeres con baja sensibilidad a la respuesta genital. A su vez, la satisfacción sexual se incrementó al final del estudio, y pasó del $65,78 \%$ a $94,73 \%(p<0,001)$, y el dolor disminuyó del $16,22 \%$ al $5,26 \%(p<0,001)$.

En este estudio, los resultados evidencian un incremento en la frecuencia sexual mensual, que se asocia a una franca mejoría en la función sexual de las mujeres participantes; esto refleja una importante elevación en la satisfacción con el tratamiento, así como con la autoconfianza generada por el tadalafilo en la mujer.

En las mujeres que tenían relaciones sexuales por iniciativa propia, en comparación con las mujeres que tenían actividad sexual por insistencia de la pareja, se observó un significativo aumento en la puntuación del IFSF, hallazgo similar a lo encontrado en un estudio publicado por Espitia-De La $\mathrm{Hoz}^{28}$ en mujeres en climaterio en el Quindío (Colombia).

La ausencia de publicaciones sobre el tratamiento, con tadalafilo, del deseo sexual hipoactivo y la excitación/lubricación en mujeres postmenopáusicas dificulta su comparación con otros estudios; sin embargo, en nuestro medio, la ausencia de patrocinadores se constituye en un problema para este tipo de investigaciones.

La evidencia respalda que las disfunciones sexuales sean abordadas con un enfoque biopsicosocial, por medio de un modelo integrador ${ }^{29}$ según corresponda: biológico, psicológico, interpersonal, sociocultural y de pareja/ vínculo, asumiéndolo no como un problema individual, sino de pareja, a fin de lograr intervenir a la dupla, en la búsqueda de obtener mayor impacto con la terapia, logrando la franca mejoría y normalización de la función sexual.

Una de las limitaciones de la presente investigación fue que se buscaba evaluar el impacto de una intervención 
farmacológica sobre la función sexual de la mujer, en la que se involucran sentimientos $\mathrm{y}$ otros aspectos subjetivos, pudiéndose presentar sesgos en los resultados. Adicional a ello, no se cuenta con datos precisos sobre las dosis efectivas del tadalafilo en mujeres, ni tampoco se controló la dosificación óptima recibida por cada una de las participantes, sino que se empleó una sola dosis para todas, lo cual podría influir en las diferencias en los resultados y en la aparición de efectos adversos. Al no tener un grupo control, se hace imposible diferenciar los efectos específicos del tratamiento.

En cuanto a las fortalezas, se destaca que es el primer estudio de estas características que se realiza en el país. Segundo, el significativo número de la muestra. Tercero, el instrumento empleado (IFSF) ha sido validado en Colombia, ${ }^{15}$ con características psicométricas adecuadas.

Una importante contribución de esta investigación consiste en la oportunidad de ofrecer alternativas terapéuticas - desde la práctica clínica diaria del especialista en ginecología -a las mujeres postmenopáusicas que presenten disfunciones sexuales y que buscan algún tipo de ayuda o tratamiento.

De esta investigación surge la necesidad de adelantar estudios con el tadalafilo en mujeres con disfunciones sexuales que presenten diferentes patologías crónicas, mediante ensayos clínicos aleatorizados y controlados.

En futuras investigaciones, se debe avanzar en el conocimiento del efecto del tadalafilo en mujeres postmenopáusicas que reciban terapia de reemplazo hormonal, para diferenciarlas de las que no la reciben, puesto que la experiencia con inhibidores de FDE-5, en terapia combinada para el tratamiento de las disfunciones sexuales femeninas es limitada pero promisoria.

\section{Conclusiones}

Las disfunciones sexuales femeninas se comprenden menos y se investigan menos; por ello, debido a su complejidad, se hace conveniente un enfoque multidisciplinario, en el que se aborden los aspectos hormonales, neurobiológicos, vasoactivos y psicosocioculturales, más allá de los potenciales efectos benéficos de un medicamento.

En este estudio, se informan resultados positivos con el tadalafilo; por lo tanto, consideramos que los IFDE5 podrían ser una alternativa terapéutica efectiva en el tratamiento de las disfunciones sexuales femeninas; por eso, invitamos a realizar mayores esfuerzos para comprender su fisiopatología, y, de este modo, desarrollar tratamientos farmacológicos eficaces para la misma.

El establecimiento y el uso de guías de manejo o protocolos puede proporcionar un enfoque integral y eficaz para abordar y tratar las disfunciones sexuales femeninas.

\section{Financiación}

Este estudio fue financiado con recursos propios del autor, los cuales fueron obtenidos de sus turnos en obstetricia.

\section{Agradecimientos}

El auto agradece a todos los integrantes del equipo de investigación, así como a las directivas de Hathor, Clínica Sexológica, y, en especial, a las mujeres participantes. También agradece la colaboración de Claudia Espitia Gallego, por el incondicional apoyo y su importante papel al motivar la realización de este proyecto.

\section{Responsabilidades Éticas}

Protección de personas y animales: el autor declara que los procedimientos seguidos se conformaron a las normas éticas del comité de experimentación humana responsable y están de acuerdo con la Asociación Médica Mundial y la Declaración de Helsinki.

Confidencialidad de los datos: el autor declara que ha seguido los protocolos de su centro de trabajo sobre la publicación de datos de pacientes.

Derecho a la privacidad y consentimiento informado: el autor ha obtenido el consentimiento informado de los pacientes y/o sujetos referidos en el artículo. Este documento obra en poder del autor de correspondencia.

Conflicto de Intereses

El autor no tiene conflicto de intereses que declarar.

\section{Referencias}

1 Blümel J. Disfunciones sexuales en latinoamericanas. Santiago, Chile: ed. Sexo. Sexualidad; 2010

2 Vizcaíno Alonso M, Montero Vizcaíno Y. Disfunciones sexuales femeninas y factores psicosociales asociados en un grupo de mujeres supuestamente sanas. Revista Sexología y Sociedad. 2017;23(02):182-194

3 Espitia-De La Hoz FJ. Prevalence and characterisation of sexual dysfunctions in women, in 12 Colombian cities, 2009-2016. Rev Colomb Obstet Ginecol 2018;69(01):9-21. Doi: 10.18597/ rcog. 3035

4 Espitia-De La Hoz FJ, Gallego-Orozco H, Gallego-Becerra HA. Comparison of the effectiveness of bupropion versus buspirone in the treatment of hypoactive sexual desire in women, a randomized clinical trial. Int J Fam Commun Med 2018;2(05): 340-344. Doi: 10.15406/ijfcm.2018.02.00105

5 Halpin DMG. ABCD of the phosphodiesterase family: interaction and differential activity in COPD. Int J Chron Obstruct Pulmon Dis 2008;3(04):543-561. Doi: 10.2147/copd.s1761

6 Nagendran J, Archer SL, Soliman D, et al. Phosphodiesterase type 5 is highly expressed in the hypertrophied human right ventricle, and acute inhibition of phosphodiesterase type 5 improves contractility. Circulation 2007;116(03):238-248. Doi: 10.1161/ CIRCULATIONAHA.106.655266

7 Wespes E, Amar E, Hatzichristou D, et al; EAU. EAU Guidelines on erectile dysfunction: an update. Eur Urol 2006;49(05):806-815. Doi: 10.1016/j.eururo.2006.01.028

8 Doumas M, Lazaridis A, Katsiki N, Athyros V. PDE-5 inhibitors: clinical points. Curr Drug Targets 2015;16(05):420-426. Doi: $10.2174 / 1389450115666141111111301$

9 Hallén K, Wiklund NP, Gustafsson LE. Inhibitors of phosphodiesterase. Br J Pharmacol 2006;(S1):147

10 Harlow SD, Gass M, Hall JE, et al; STRAW 10 Collaborative Group. Executive summary of the Stages of Reproductive Aging Workshop +10 : addressing the unfinished agenda of staging reproductive aging. Menopause 2012;19(04):387-395. Doi: $10.1097 / g m e .0 b 013 e 31824 d 8 f 40$ 
11 Espitia-De La Hoz FJ. Prevalence of genitourinary syndrome of menopause and impact on sexuality of women in Quindío (Colombia), 2013-2016. Rev Colomb Obstet Ginecol 2018;69 (04):249-259. Doi: 10.18597/rcog.3111

12 Giraldi A, Rellini AH, Pfaus J, Laan E. Female sexual arousal disorders. J Sex Med 2013;10(01):58-73. Doi: 10.1111/j.17436109.2012.02820.x

13 Porst H, Giuliano F, Glina S, et al. Evaluation of the efficacy and safety of once-a-day dosing of tadalafil $5 \mathrm{mg}$ and $10 \mathrm{mg}$ in the treatment of erectile dysfunction: results of a multicenter, randomized, double-blind, placebo-controlled trial. Eur Urol 2006;50(02):351-359. Doi: 10.1016/j.eururo.2006.02.052

14 Rosen R, Brown C, Heiman J, et al. The Female Sexual Function Index (FSFI): a multidimensional self-report instrument for the assessment of female sexual function. J Sex Marital Ther 2000;26 (02):191-208. Doi: 10.1080/009262300278597

15 Vallejo-Medina P, Pérez-Durán C, Saavedra-Roa A. Translation, adaptation, and preliminary validation of the female sexual function index into spanish (Colombia). Arch Sex Behav 2017; ..*:1-14. Doi: 10.1007/s10508-017-0976-7

16 Espitia De La Hoz FJ. Evaluación de la prevalencia de disfunción sexual en mujeres medicos, del Eje Cafetero colombiano, en etapa de climaterio. Archivos de Medicina (Col) 2017;17:70-77

17 Sand M, Rosen R, Meston C, Brotto LA. The female sexual function index (FSFI): a potential "gold standard" measure for assessing therapeutically-induced change in female sexual function. Fertil Steril 2009;92(03):S129. Doi: 10.1016/j.fertnstert.2009.07.1173

18 Zhang $\mathrm{WH}$, Zhang $\mathrm{XH}$. Clinical and preclinical treatment of urologic diseases with phosphodiesterase isoenzymes 5 inhibitors: an update. Asian J Androl 2016;18(05):723-731. Doi: 10.4103/1008-682X.167721

19 Andersson K-E, Nomiya M, Sawada N, Yamaguchi O. Pharmacological treatment of chronic pelvic ischemia. Ther Adv Urol 2014;6(03):105-114. Doi: 10.1177/1756287214526768

20 Jansson PA, Murdolo G, Sjögren L, et al. Tadalafil increases muscle capillary recruitment and forearm glucose uptake in women with type 2 diabetes. Diabetologia 2010;53(10):2205-2208. Doi: 10.1007/s00125-010-1819-4
21 Rusiecki J, Rao Y, Cleveland J, Rhinehart Z, Champion HC, Mathier MA. Sex and menopause differences in response to tadalafil: 6minute walk distance and time to clinical worsening. Pulm Circ 2015;5(04):701-706. Doi: 10.1086/683829

22 Nappi RE. Why are there no FDA-approved treatments for female sexual dysfunction? Expert Opin Pharmacother 2015;16(12): 1735-1738. Doi: 10.1517/14656566.2015.1064393

23 Caruso S, Cicero C, Romano M, Lo Presti L, Ventura B, Malandrino C. Tadalafil $5 \mathrm{mg}$ daily treatment for type 1 diabetic premenopausal women affected by sexual genital arousal disorder. J Sex Med 2012;9(08):2057-2065. Doi: 10.1111/ j.1743-6109.2012. 02777.x

24 Ashton AK, Weinstein W. Tadalafil reversal of sexual dysfunction caused by serotonin enhancing medications in women. J Sex Marital Ther 2006;32(01):1-3. Doi: 10.1080/00926230 500229079

25 Eardley I, Cartledge J. Tadalafil (Cialis) for men with erectile dysfunction. Int J Clin Pract 2002;56(04):300-304

26 Espitia-De La Hoz FJ, Orozco-Santiago L. Eficacia de dos inhibidores de la fosfodiesterasa 5 combinados con ginkgo biloba en la disfunción eréctil en hombres con enfermedades crónicas. Revista Colombiana de Endocrinología Diabetes \& Metab 2019;6(03):155-163

27 Gao L, Yang L, Qian S, Li T, Han P, Yuan J. Systematic review and meta-analysis of phosphodiesterase type 5 inhibitors for the treatment of female sexual dysfunction. Int J Gynaecol Obstet 2016;133(02):139-145. Doi: 10.1016/j.ijgo.2015. 08.015

28 Espitia-De La Hoz FJ. Menopause and sexuality: characterization of sexual dysfunctions during climacteric, in women of Quindío (Colombia). Obstet Gynecol Int J 2019;10(06):419-424. Doi: 10.15406/ogij.2019.10.00477

29 Althof SE, Leiblum SR, Chevret-Measson M, et al. Psychological and interpersonal dimensions of sexual function and dysfunction. J Sex Med 2005;2(06):793-800. Doi: 10.1111/j.1743-6109.2005. 00145.x 